\title{
Lettre inédite de Madame Cottin sur la loi du divorce
}

Silvia Lorusso

\section{(2) OpenEdition}

Journals

Édition électronique

URL : http://journals.openedition.org/rief/988

DOI : $10.4000 /$ rief.988

ISSN : 2240-7456

Éditeur

Seminario di filologia francese

Référence électronique

Silvia Lorusso, "Lettre inédite de Madame Cottin sur la loi du divorce », Revue italienne d'études françaises [En ligne], 1 | 2011, mis en ligne le 15 décembre 2011, consulté le 01 mai 2019. URL : http:// journals.openedition.org/rief/988; DOI : 10.4000/rief.988

Ce document a été généré automatiquement le 1 mai 2019.

\section{(c) (i) (9)}

Les contenus de la RIEF sont mis à disposition selon les termes de la Licence Creative Commons Attribution - Pas d'Utilisation Commerciale - Pas de Modification 4.0 International. 


\title{
Lettre inédite de Madame Cottin sur la loi du divorce
}

\author{
Silvia Lorusso
}

1 Les informations que nous avons sur la vie de Marie Sophie Cottin, née Risteau, sont encore fragmentaires et incertaines. Nous savons qu'elle est née à Paris en 1770 ; qu'en 1789 elle a épousé, et c'est un mariage d'amour, Jean-Paul-Marie Cottin ; qu'elle est restée veuve à 23 ans ; qu'elle est morte de maladie en 1807. Mais surtout, elle a écrit entre 1799 et 1806 cinq romans sentimentaux qui furent des best-sellers à l'époque et un petit poème ${ }^{1}$. Sa correspondance, presque entièrement inédite, confirme qu'elle a été une femme avec des idées non communes pour l'époque et une sensibilité que l'on pourrait définir moderne.

2 La lettre transcrite ici, envoyée par Mme Cottin à son cousin Lemarcis après la mort de sa femme $^{2}$, comprend deux parties : la première sur une polémique d'actualité, la deuxième sur une question privée. Mais toutes deux traitent du même argument : le mariage. Mme Cottin y soutient le droit à sa dissolubilité légale par le divorce et la nécessité de chacun de continuer sa vie après la mort de l'époux ou de l'épouse. Deux conditions différentes où l'on reconnait les limites des pouvoirs du mariage.

3 La lettre n'est pas datée. Mais il n'est pas difficile de la situer grâce à sa référence au livre de Mme Necker (Suzanne Curchod, 1737-1794), Réflexions sur le divorce, paru dans la seconde moitié de 1794, après la mort de l'auteur, le 15 mai 1794. Donc cette lettre a été écrite à l'automne de 1794.

4 Le 20 septembre 1792 la loi avait décrété la laïcisation de l'état civil et l'autorisation du divorce par consentement, pour cause d'incompatibilité d'humeur et de caractère, pour cause déterminée. Avec le décret du 8 nivôse an II (28 décembre 1793), la Convention avait diminué le délai nécessaire entre le divorce et le remariage. Enfin les décrets des 4-9 floréal an II (23-28 avril 1794) avaient déclaré légaux les divorces prononcés avant la loi du 20 septembre 1792 : il suffisait d'une séparation de fait de six mois entre les époux. Dans son pamphlet, Mme Necker se déclare ennemie du divorce, procédure qui détruirait les quatre buts que poursuit le mariage: «le bonheur individuel des Epoux dans la 
jeunesse »; le bonheur des enfants ; « la pureté des mœurs » (qui serait un «but manqué par le divorce ») ; « la consolation, le secours, et quelquefois le bonheur de la vieillesse». Elle revendique le fait que « les oppositions de caractère ne sont pas invincibles, comme les résistances de l'instinct animal $»^{3}$ et que l'habitude peut rapprocher des caractères opposés. Quant aux fils : « une bonne éducation déjà si difficile pour les parents les plus unis, devient impossible sous la loi du divorce $»^{4}$. Elle reconnaît qu'il y a des femmes qui, grâce au divorce, pourraient se dérober à la tyrannie de leur mari, mais les lois ne sont pas faites pour les exceptions. Selon Jésus-Christ le divorce et l'adultère sont synonymes et les Grecs font dériver le crime du vice. Donc « la permission du divorce est contraire aux nobles et pures institutions de la nature $»^{5}$. Et le mariage est la seule consolation qui nous reste dans la vieillesse.

Mme Cottin est d'un avis tout à fait contraire : le divorce ne corrompt pas les mœurs, mais il se révèle un remède pour essayer de rendre plus forte la morale de la Nation. La question a été mal posée par Mme Necker qui parle d'un monde imaginaire, habité par des personnes comme elles devraient être et non pas comme elles sont. La romancière définit avec mépris « un roman » le pamphlet de Mme Necker.

Mme Cottin avait déjà défendu le divorce dans une autre lettre (commencée par son mari), écrite en 1790 et adressée à sa cousine et confidente Julie Verdier :

Je veux te dire et te demander si tu est [sic] de mon avis sur l'approbation que je donne au divorce qu'il paraît que l'assemblée nationale veut établir en France, selon mon idée ce sera un grand bien, comme je pense que l'état de mariage ne peut être qu'un très heureux ou très malheureux, il est bien juste que ceux qui sont dans cette dernière position ne soient pas destinés à y passer toute leur vie, ou à désirer pour en changer, la destruction d'un être dont l'existence est un obstacle à la félicité.[Lettre de Jean-Paul Cottin à Julie Venès-Verdier, BnF, NAF 15976, fo 96]

7 En revendiquant la nécessité du divorce, Sophie ne pensait pas à sa situation parce qu'elle aimait tendrement son mari. À la différence de Mme Necker, elle ne considérait pas le mariage comme un état de nature, mais comme une «institution sociale»: donc, le cas échéant, révocable [f' 95]. Dans cette même lettre elle répond aussi à une des critiques habituelles des opposants du divorce qui sera reprise également par Mme Necker :

J'approuve d'autant plus le divorce qu'en l'établissant on [n']oublie pas les malheureux fruits d'une union désassortie, dans le contrat de mariage on fixe un sort pour les enfants en cas de divorce de sorte que [...] ils sont au moins à l'abri de la fortune. [f० 96]

D'ailleurs dans sa jeunesse déjà, elle n'avait pas une idée romanesque sur le mariage, comme l'atteste la lettre à Julie Verdier, non datée mais que l'on peut considérer écrite entre 1785 et 1788 , donc avant son mariage :

Je sais bien que c'est une idée fausse que d'imaginer que le mariage est un enfer s'il n'est pas un paradis, il n'est presque jamais ni l'un ni l'autre, son état habituel est un attachement modéré, fondé sur [...] la communauté de biens, les enfants et l'habitude, cela forme un tout assez doux qui compense l'humeur qu'occasionne le désassortiment des goûts et l'opposition de l'humeur. [Lettre de Sophie Cottin à Julie Venès-Verdier, BnF, NAF 15970, $\mathrm{f}^{\circ}$ 4]

Dans la deuxième partie de la lettre présentée ici, Mme Cottin exprime sa compassion face à la douleur inexprimable de son cousin pour la mort de sa femme. Mais elle lui conteste son désir de ne plus vivre, sinon dans le souvenir de l'aimée. Elle désapprouve en particulier son aspiration à prendre dans la vie la place de la morte, à «la copier » : à penser et à agir comme elle pour essayer de la sentir encore à ses côtés. Sophie constate avec une lucidité surprenante un protocole pathologique d'élaboration du deuil que 
Freud décrira dans son célèbre essai, Deuil et Mélancolie (1917) : elle réprouve que ne pouvant plus avoir la femme aimée, il essaye d'être celle-ci, de substituer l'être à l'avoir. Elle fait allusion avec délicatesse au Temps qui a le pouvoir de guérir les blessures. En effet son cousin se remariera.

Enfin il faut observer que dans ces lettres Sophie traite le mariage d'une façon très différente par rapport aux romans de femme de l'époque (y compris les siens) qui n'admettent pas le divorce du fait que l'on y considère volontiers comme impossible la séparation, même après la mort.

11 J'ai modernisé l'orthographe de la lettre. La graphie de Mme Cottin, plutôt claire, suit les usages de l'orthographe du temps (parens, tems ...). L'abolition des accents, des tirets et de la ponctuation, les contractions des mots (quelle, quils, peutetre, saisje...) lui viennent du style épistolaire privé. La ponctuation, respectée autant que possible, a été insérée et adaptée à l'usage moderne afin de rendre le texte compréhensible. Dans trois cas j'ai conservé la version originale qui présente des fautes de concordance, ou dans l'emploi des modes verbaux, indiquées par [sic].

\section{ANNEXES}

Des jolies idées et pas une seule bonne raison, voilà ce que j'ai trouvé dans l'ouvrage de Mme Necker, et ce n'est pas ainsi qu'on doit traiter une question aussi importante que celle du divorce ; elle est partie d'un principe faux et qui renverse du premier abord tous les motifs qu'elle peut alléguer. Elle envisage les hommes tels qu'ils devraient être, au lieu de les voir tels qu'ils sont, et cette erreur funeste, dont j'ai éprouvé plus que personne les pernicieux effets, fait de son livre un roman agréable et non pas un écrit utile. Ne raisonnons point et regardons autour de nous : dans la Hollande et autres pays où le divorce est permis, les mœurs sont-elles moins respectées ? et en France avant que cette loi fut établie, quand ce joug de l'indissolubilité pesait sur le mariage, n'étions-nous pas inondés de mauvais ménages, de femmes infidèles, d'éclats scandaleux, d'enfants ingrats, de mauvais pères? jamais les mœurs furent-elles plus corrompues que durant les années qui ont précédé la loi du divorce, n'est-ce pas même l'excès de la dépravation qui l'a provoquée? on ne vous la donne pas comme une bonne chose, mais comme un remède ; on ne vous la donne pas pour rendre les femmes honnêtes, mais pour les empêcher de joindre la fourberie et le mensonge, au dérèglement des mœurs. Mettez-nous dans le monde idéal que peint Mme Necker et je serai de son avis, mais où est-il ce monde ? dans la tête des Etres jeunes, bons et ardents, peut-être y ai-je cru comme un autre, mais cette illusion a fui avec toutes celles de la jeunesse, et je ne comprends pas que Mme Necker l'ait conservée si longtemps. J'ai été tentée de prendre la plume pour la réfuter d'un bout à l'autre, mais les raisons qu'elle alleguent [sic] n'en valent pas la peine, elles ne tromperont que ceux qui veulent l'être, et ceux-là, il n'y a rien à leur dire... Vous êtes du nombre, mon cousin, je vous vois avec effroi sur un chemin où j'ai été longtemps ; l'excès du malheur sous lequel vous gémissez exalte l'imagination; quand on voit ce qu'on aime parmi les anges, on n'aspire qu'à leur ressembler, les vertus les plus sévères ne sont plus 
des sacrifices et la perfection parait possible ; mais la perfection, cette dangereuse chimère, est incompatible avec notre nature, toutes nos facultés ont beau tendre vers elle, elles ne pourront jamais y arriver, et en nous fixant sur un but impossible nous risquons de nous éloigner de la nature et de la vérité. On y revient/ [ $\mathrm{f}^{\circ} 6$ ] enfin parce qu'elles ont une force à laquelle on ne résiste pas, mais qui peut nombrer les malheurs que l'exagération des vertus factices a pu causer ?... mais à quoi bon vous dire tout cela, ne sais-je pas que l'expérience des autres n'est qu'un vain bruit et qu'on ne croit qu'à la sienne ? ... mon cousin vous avez deux âmes en vous, d'abord celle de Mme Lemarcis, et puis la vôtre qui garde le silence. Vous ne suivez point vos opinons, vous n'adoptez que celles qu'elle aurait eues, vos sensations même sont étouffées pour y substituer celles qu'elle aurait éprouvées ; c'est mieux que vous-même diriez-vous ; oui je crois en effet qu'elle valait mieux que vous, mais en la calquant, vous n'êtes ni elle ni vous, et vous êtes au-dessous de tous deux. Ces caractères copiés ressemblent à ces pièces de rapport qui, jolies séparément, n'ont ni unité ni ensemble, et en effet qui peut remplacer ce charme aussi aimable que la vertu même, le naturel ? Être soi, toujours soi, voilà ce qui touche, voilà ce qui entraîne, voilà ce qui donnait sans doute un attrait si puissant aux vertus de votre amie, et voilà ce qu'on perd en imitant. Aimez-la, parlez d'elle avec votre âme, jugez avec votre esprit, sentez avec votre cœur ; elle le préférait [sic] sans doute car vous vaudriez mieux ainsi. Vos vertus alors plus solides seraient à vous, et ne tiendraient pas à un sentiment que le temps... je me tais, il est des cordes sensibles qu'une main étrangère ne doit pas toucher. J'en ai peut-être beaucoup trop dit, j'ai peut-être blessé des sensations délicates et tendres et fait évanouir cette aurore d'amitié dont vous me parliez hier, mais enfin pour la développer ou l'éteindre, il faut que vous me connaissiez, et je ne sais d'autre moyen pour cela que de dire ma pensée!

\section{NOTES}

1. Claire d'Albe (1799); Malvina (1801); Amélie Mansfield (1802); Mathilde (1805); Elisabeth, ou les Exilés de Sibérie (1806). Et le petit poème en prose La Prise de Jéricho, ou La Pécheresse convertie paru pour la première fois dans Les Mélanges de Suard en 1803.

2. Lettre à Lemarcis, dans Papiers de Mme Cottin, XXVI, Lettres de divers correspondants à Sophie Cottin , fo 1-334, BnF, NAF 15984, f ${ }^{\circ}$ 5-6.

3. Réflexions sur le divorce, par Mad. Necker, Lausanne, Durand Ravanel et Comp, 1794, p. 10.

4. Ibid., p. 47.

5. Ibid., p. 73.

\section{INDEX}

Mots-clés : divorce, féminisme, Révolution, Mme Cottin, Mme Necker 